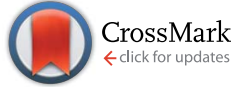

Cite this: RSC Adv., 2015, 5, 98834

Received 16th July 2015

Accepted 10th November 2015

DOI: $10.1039 / \mathrm{c} 5 \mathrm{ra14076e}$

www.rsc.org/advances

\title{
The Maya blue nanostructured material concept applied to colouring geopolymers $\uparrow$
}

\author{
Claudiane Ouellet-Plamondon, $\xi^{\star a}$ Pilar Aranda, ${ }^{\mathrm{b}}$ Aurélie Favier, ${ }^{\mathrm{c}}$ Guillaume Habert, ${ }^{\mathrm{a}}$ \\ Henri van Damme ${ }^{d}$ and Eduardo Ruiz-Hitzky ${ }^{b}$
}

Maya blue is an ancient nanostructured pigment synthetized by assembling indigo, a natural dye, with palygorskite, a microfibrous clay mineral. The novelty of our approach is to mimic "pre-Columbian nanotechnology" and to functionalize geopolymers with a sepiolite-based hybrid organic-inorganic nanocomposite inspired from the Maya blue. It is acid- and UV-resistant, as confirmed by the stability of Maya mural paintings over time. We synthesized analogous pigments, using methylene blue (MB) and methyl red (MR) as organic dyes and sepiolite as fibrous clay mineral. We used an aqueous and a solidstate method, both leading to encapsulation of dye monomers into the clay micropores, as confirmed by UV-vis spectroscopy. This nanostructured pigment was then included into a geopolymer matrix at room temperature. The stability of the new material to UV and acid was tested. It was confirmed that it is the prior encapsulation of the dye into sepiolite that leads to the stability of the pigment in the geopolymer matrix. This first study opens the way to numerous possibilities for functionalizing inorganic binder materials with organic elements that would be otherwise sensitive to thermal treatment in conventional ceramic processing.

\section{Introduction}

Several centuries ago, people from Mesoamerica used an amazing pigment of extraordinary thermal and chemical stability as it resists temperatures up to about $250^{\circ} \mathrm{C}$, exposure to strong concentrated acids and alkalis, as well as organic solvents. ${ }^{1,2}$ This pigment, known as Maya blue, was most commonly used during the late pre-Spanish period in both pottery and temple murals, probably as early as the 6th century AD. ${ }^{3}$ Later-on, its use was pursued in Spain during the 16th and 17th centuries and until the 19th century in Cuba under the Havana blue name., ${ }^{4,5}$ It is now known that the Maya blue pigment is actually an ancient nanostructured material formed by assembling indigo, a natural dye, with palygorskite, a microfibrous clay mineral..$^{6-8}$ Due to its exceptional stability, Maya blue was first considered to be an entirely inorganic

${ }^{a}$ Institute for Construction and Infrastructure Management, Chair of Sustainable Construction, ETH Zurich, Switzerland. E-mail: Claudiane.Ouellet-Plamondon@ etsmtl.ca

${ }^{b}$ Materials Science Institute of Madrid, CSIC, c/ Sor Juana Inés de la Cruz 3, 28049 Madrid, Spain

${ }^{c}$ Laboratory of Construction Materials, EPFL Lausanne, Switzerland

${ }^{d}$ Department of Civil \& Environmental Engineering, MIT, and $<M S E 2>$, The Joint MITCNRS Unit, 77 Massachusetts Avenue, Room 1-278, Cambridge, MA 02139-4307, USA $\dagger$ Electronic supplementary information (ESI) available. See DOI: 10.1039/c5ra14076e

\$ Present address: Department of Construction Engineering, École de Technologie Supérieure, 1100 Notre-Dame West, Montréal (Québec), H3C 1K3, Canada. pigment, ${ }^{3}$ but it was soon recognized that it is actually an organic-inorganic nanohybrid material, in which the organic dye molecules are encapsulated within the structural nano sized tunnels of palygorskite. ${ }^{2,9,10}$ This ultraconfined environment is stabilizes the dye, preventing its chemical, thermal, and biological degradation.

Palygorskite, also known as attapulgite, is a hydrated magnesium and aluminium silicate of ideal formula $\mathrm{Si}_{8} \mathrm{O}_{20^{-}}$ $\mathrm{Mg}_{5}(\mathrm{OH})_{2}\left(\mathrm{H}_{2} \mathrm{O}\right)_{4} \cdot 4 \mathrm{H}_{2} \mathrm{O}^{.11}$ Its structure consists of alternating blocks composed by two tetrahedral sheets of silica layers sandwiching a central octahedral layer of magnesium, aluminium and, to a minor extent, other ions present as isomorphous substitutions. Such alternate organization of blocks determines the presence of structural of nanometric dimensions $(0.64 \times 0.37 \mathrm{~nm})$, growing along the fibre direction ( $c$ axis). Sepiolite, another microfibrous clay mineral parent to palygorskite, has also been found in some Maya blue samples. It is a hydrous magnesium silicate of ideal formula $\mathrm{Si}_{12} \mathrm{O}_{30} \mathrm{Mg}_{8^{-}}$ $(\mathrm{OH})_{4}\left(\mathrm{H}_{2} \mathrm{O}\right)_{4} \cdot 8 \mathrm{H}_{2} \mathrm{O},{ }^{11}$ showing a structural arrangement akin to palygorskite. Its tunnels have a slightly larger cross-section $(1.06 \times 0.37 \mathrm{~nm})$ than those of palygorskite. Interestingly, these structural cavities act as micropores, able to adsorb atoms, molecules, and even polymers. ${ }^{12}$ In addition, the external surface of the fibers is covered by silanol groups (Si-OH) that may interact with a great variety of compounds, leading to new architectures and nanocomposite materials useful for advanced applications. ${ }^{13-15}$ The strong bonding between the clay and the indigo molecules in Maya blue was 
first related to these $\mathrm{OH}$-covered external surfaces. ${ }^{15}$ Later on, other authors ${ }^{\mathbf{1 6}}$ proposed that the strong fixation of indigo molecules can be ascribed to their penetrating into the clay tunnels. Actually, as pointed out by Arnold et al., ${ }^{16}$ it appears that various bonding scenarios might exist, depending on the preparation technique. , $^{\mathbf{8 1 7}, \mathbf{1 8}}$

The accessibility of different molecular dyes to palygorskite and sepiolite fibrous clays opens the way to the preparation of a wide variety of synthetic Maya blue analogs. For instance, it has been shown that methylene blue in water solution adsorbs on sepiolite with a partial or even deep penetration into the structural tunnels (Fig. 1). ${ }^{\mathbf{1 9 - 2 1}}$ This has led to the development of Maya blue-like pigments for colouring durably the polymer matrix in polymer-clay nanocomposites. ${ }^{22}$ In the present paper, we intend to take advantage of the exceptional chemical stability of Maya blue-like pigments for colouring a class of promising inorganic binders known as geopolymers. Due to their lower carbon footprint, geopolymers are considered as possible substitutes to Portland cement, the manufacturing of which is responsible for an estimated $7 \%$ of total anthropogenic carbon dioxide emissions. ${ }^{23}$ Geopolymers are formed by activation of a solid aluminosilicate source in a strongly alkaline solution. The reaction leads to a highly amorphous material with a low calcium content and, concomitantly, to a very rapid solidification of the slurry and early strength development. ${ }^{24,25}$ The geopolymer network is composed of cross-linked Si-O-Al$\mathrm{O}-\mathrm{Si}$ chains in which the aluminium ions are in tetrahedral coordination. Each aluminium ion is associated with an alkaline cation in order to compensate for the charge deficit introduced by the Al-for-Si substitution. Geopolymers are not only promising alternative materials to Portland cement in term of environmental impacts. ${ }^{26}$ They are also well known for their better acid $^{27}$ and fire resistance ${ }^{28}$ compared to Portland cementbased materials. However, just like a Portland cement mortar or concrete, a fresh geopolymer slurry is a very aggressive medium, due to the very high $\mathrm{pH}$ of the solution and early attempts to introduce acid-base dye indicators, such as methyl red, in geopolymers experienced severe stability problems, presumably due to the lack of protection of the dye molecules. ${ }^{29}$ The novelty of our approach is to mimic "pre-Columbian nanotechnology"

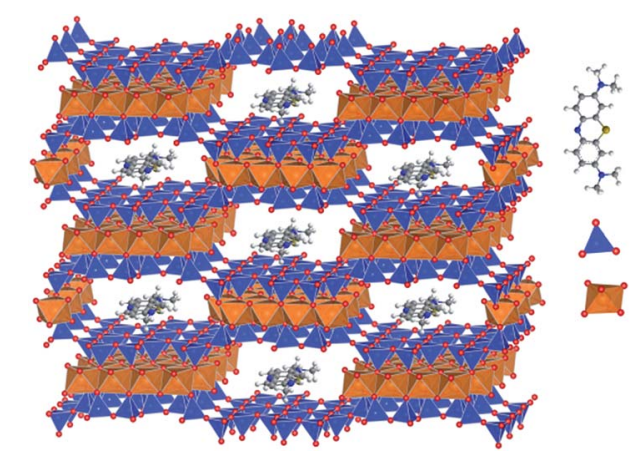

Fig. $1 \mathrm{MB}$ incorporation along the sepiolite tunnels (left), with individual constituents depicted at the right: MB molecule (blue: $N$, gray: C, yellow: S, white: $\mathrm{H}$ ), silicon oxide tetrahedrum (blue: si, red: O) and magnesium oxide octahedrum (orange: $\mathrm{Mg}$, red: O). to functionalize geopolymers with a sepiolite-based hybrid organic-inorganic nanocomposite inspired from the Maya blue. In the present work, we have centered our interest to show the role of sepiolite in the encapsulation of organic dyes for their protection towards light and external reagents.

\section{Experimental}

\subsection{Materials}

The sepiolite clay was a high purity and micronized product of rheological grade, obtained from Vallecas (Madrid) deposits and commercialized as Pangel S9, by TOLSA SA (Madrid, Spain). Methylene blue ( $\mathrm{MB}, \mathrm{C}_{16} \mathrm{H}_{18} \mathrm{ClN}_{3} \mathrm{~S}$ ) was ordered from Acros Organics and methyl red $\left(\mathrm{C}_{15} \mathrm{H}_{15} \mathrm{~N}_{3} \mathrm{O}_{2}\right)$ reagent of $95 \%$ purity present as sodium salt ( $\mathrm{MR}, \mathrm{NaC}_{15} \mathrm{H}_{14} \mathrm{~N}_{3} \mathrm{O}_{2}$ ) was obtained from Sigma Aldrich. The activating solution for geopolymer synthesis was prepared with $\mathrm{SiO}_{2}$ (Merk), $\mathrm{K}_{2} \mathrm{O}$ (Chemie Brunschwig AG) and demineralized water. A commercial Metakaolin (Metastar 501 ) containing less than $5 \%$ of quartz impurities was obtained from Imerys $\left(\mathrm{SiO}_{2}: 50.44 \%, \mathrm{Al}_{2} \mathrm{O}_{3}: 43.92 \%, \mathrm{TiO}_{2}: 1.00 \%, \mathrm{Fe}_{2} \mathrm{O}_{3}\right.$ : 0.46\%, MgO: 0.09\%, $\left.\mathrm{NaO}: 0.15 \%, \mathrm{SO}_{3}: 0.19, \mathrm{Cl}: 0.01 \%\right)$. A quartz sand fraction of size $0.1-0.6 \mathrm{~mm}$ was used for preparation of the mortars.

\subsection{Pigments synthesis}

The pigments were prepared by two methods, either from MB or MR aqueous solutions (products referred to as "as"), or by solid state reaction between the clay and the dye (products referred to as "ss"), respectively. For the aqueous method, one gram of sepiolite was stirred for at least 12 hours in $100 \mathrm{ml}$ of $0.0005 \mathrm{M}$ solution of MB or MR conditions, in which is the dye is present as monomers. ${ }^{19}$ The resulting solid was then extracted by centrifugation and rinsed with water until the supernatant was clear. In the solid state method, $8 \%$ by weight of dye was manually mixed with the sepiolite clay using a mortar and pestle. ${ }^{21}$ The mixture was then heated for 20 minutes at $250{ }^{\circ} \mathrm{C}^{21}$ The resulting sepiolite-based pigments are shown in Fig. 2. Note that no washing step has been done and therefore, even if other studies have followed the same protocol, ${ }^{22}$ an excess of pigment cannot be exclude.

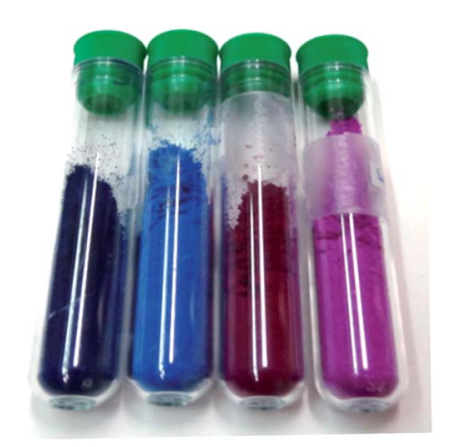

Fig. 2 Resulting sepiolite pigment: $M B_{s s}, M B_{a s}, M R_{s s}, M R_{a s}$ (from left to right). 


\subsection{Geopolymer synthesis and sample preparation}

The composition of the activating solution was $\mathrm{SiO}_{2} / \mathrm{K}_{2} \mathrm{O}=1.05$ and $\mathrm{H}_{2} \mathrm{O} / \mathrm{K}_{2} \mathrm{O}=11$ ( $\mathrm{pH} 14.6$, density $1.44 \mathrm{~g} \mathrm{ml}^{-1}$ ). The solutions were mixed with a magnetic stirrer for $24 \mathrm{~h}$ to allow for cooling and species equilibration. The geopolymer samples were then made by mixing the activating solution with high quality metakaolin in order to achieve the following molar ratios in the final product: $\mathrm{Si} / \mathrm{Al}_{2} \mathrm{O}_{3}=3$ and $\mathrm{K}_{2} \mathrm{O} / \mathrm{Al}_{2} \mathrm{O}_{3}=1$. A compressive strength of $55 \pm 2 \mathrm{MPa}$ was achieved when the geopolymer was tested on mortars made with 50 vol\% mortar sand.

To prepare the pigment-loaded geopolymers, $1.2 \%$ by weight of sepiolite/dye composite pigment was mixed with the metakaolin powder. Mixing was first done with a spatula, then with the ultraturrax T50 mixer (IKA Labortechnik) at $3000 \mathrm{rpm}$ for 3 minutes and then at higher speed (6000 rpm) for another 3 minutes in order to ensure a homogeneous mixture. The alkaline solution was then added and mixed for 3 minutes at high speed. Finally, the fine sand was incorporated on a $50 \%$ volume basis and mixed with the geopolymer paste during two periods of 2 minutes at $3000 \mathrm{rpm}$ interrupted by one minute of manual stirring as it is done conventionally in mortar preparation protocols, and then another two minutes at $3000 \mathrm{rpm}$. The mortar were then poured in a formwork and vibrated for 1 minute to remove air bubbles. The samples were kept one day in the formwork wrapped in plastic foil and aluminium foil in order to allow for endogenous curing without evaporation nor carbonation of the paste. Six to nine $3.5 \times$ $3.5 \times 3 \mathrm{~cm}^{3}$ cubes were made per batch.

Control samples were prepared by incorporating MB directly into the geopolymer matrix, without encapsulation in the sepiolite clay $(0.02 \%$ or $0.5 \%$ by weight of $\mathrm{MB}$ with respect to metakaolin). Note that $0.2 \%$ is the MB equivalent quantity when $1.2 \%$ of sepiolite composite pigment is incorporated. Another control geopolymer sample was prepared by incorporating $1 \%$ sepiolite and $0.5 \%$ MB separately in the metakaolin powder.

\subsection{Characterisation}

The geopolymer mortar and the sepiolite clay were characterized by X-ray diffraction (XRD) using a Bruker D8 T2T Advance diffractometer equipped with a $\mathrm{Cu}-\mathrm{K}_{\alpha}$ source, with a scan step of $2^{\circ}$ $\min ^{-1}$, from 2 to $70^{\circ} 2 \theta$ values. Calorimetry measurements were performed with an I-Cal 8000 instrument from Calmetrix to follow the geopolymeric reaction. To characterize the dye and the composite pigment, we performed UV-vis experiment with a UV2401 PC Shimadzu spectrometer. The measurement were taken in reflectance mode and transformed to diffuse reflectance by the Kubelka-Munk function $\left(F(R)=(1-R)^{2} / 2 R\right){ }^{30}$ The specific surface area of the samples was determined by applying the single-point BET method to the adsorption/desorption of $\mathrm{N}_{2}$ at $77 \mathrm{~K}$ (Micromeritics Flowsorb II 2300). The BET specific surface area and micropore volume of sepiolite and sepiolite-dye samples were calculated from the nitrogen adsorption-desorption isotherms obtained with a Micrometics ASAP 2010 equipment in samples outgassed at $150{ }^{\circ} \mathrm{C}$. The dye content in the sepiolite-dye synthetic pigment was calculated from the carbon content in the samples determined by CHN elemental analysis with a Perkin Elmer 2400 series II CHNS/O elemental analyzer.
The stability of the mortars in acid medium and under UV exposure was tested as follows. UV tests were conducted in a chamber with a SOL500 lamp head mounted to a UVACUBE400 with a $\mathrm{H}_{2}$ outdoor filter (Honle UV technology) at minimum exposition $(5 \mathrm{~W})$ for a $48 \mathrm{~h}$ period, and at higher exposition ( $800 \mathrm{~W}$, six times the natural sunlight) for two successive $24 \mathrm{~h}$ periods. The resistance to acid was tested in triplicates in 1\% sulphuric acid solution for $48 \mathrm{~h}$ and in a sulphuric acid solution adjusted to $\mathrm{pH} 2$ for one week. The colour was then measured with a CM-700d Konica Minolta spectrophotometer. The illuminant was CIE D65 and the viewing angle was $10^{\circ}$. The number of colour measurements was determined with a Student's $t$-test. The colour was determined by the three coordinate $L$ for the intensity, $a$ and $b$ for the chromaticity. ${ }^{31}$ The $L$ reflects the white axis; the $a$ the "red-greenness" axis and the $b$ the "yellow-blueness" axis. ${ }^{32}$ The colour difference is calculated according to:

$$
\Delta E_{a b}=\sqrt{\left(L_{2}-L_{1}\right)^{2}+\left(a_{2}-a_{1}\right)^{2}+\left(b_{2}-b_{1}\right)^{2}}
$$

where $\left(L_{1}, a_{1}, b_{1}\right)$ is the average of 15 measurements collected on three cubes that had the same treatment and $\left(L_{2}, a_{2}, b_{2}\right)$ is also an average of 15 measurements collected on three cubes with another treatment. The value of the $\Delta E$ that corresponds to the Just Noticeable Difference (JND) is a $\Delta E$ of 2.3.

\section{Results and discussion}

\subsection{Dye adsorption and encapsulation}

As illustrated in Fig. 3, the UV-vis spectra of the sepiolite-dye pigments show that the monomer form of the dye is the dominant species in the clay. Indeed, the monomer form of $\mathrm{MB}$ has a main absorption band in the $600-670 \mathrm{~nm}$ region, while
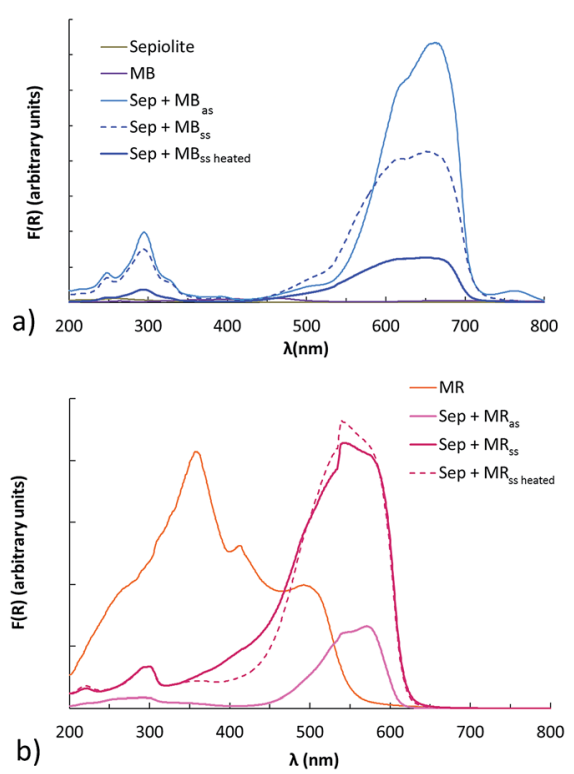

Fig. 3 UV-visible diffuse reflectance spectra of the (a) methylene blue and (b) methyl red-sepiolite synthetic pigments, prepared either by the aqueous solution method or by the solid-state method, before and after heating at $250^{\circ} \mathrm{C}$. 
dimers and higher aggregates show absorption bands centred at $570 \mathrm{~nm} .{ }^{33}$ The observed adsorption of the UV-visible spectrum of MB monomers is comparable to that observed in original Maya blue samples from archaeological sites in Mexico. ${ }^{34} \mathrm{~A}$ similar behaviour is observed with MR based pigments (Fig. 3). Interestingly, the dominance of the monomer species is observed with both preparation methods, in spite of the much higher dye content obtained with the solid state method (Table 1).

While MB and MR monomers are expected to fit into the tunnels of the sepiolite, aggregates cannot, due to steric hindrance. ${ }^{13,19}$ In agreement with this observation, the data in Table 1 show that the microporous volume and the nitrogenBET specific surface area of the clay are drastically reduced by adsorption of the dye molecules. The order of magnitude decrease is the same for both dyes and, as expected, it is related to the amount of adsorbed molecules. However, the relationship is far from being linear. The relatively small quantities of dyes introduced by the wet synthesis method induce a comparatively larger decrease of surface area and microporosity. This may be explained by the pore blocking effect of the first adsorbed molecules (two molecules at both ends of a microchannel may block the access to the entire channel space). ${ }^{12}$ In addition, when large amounts of dye molecules are adsorbed, a fraction of molecules may be adsorbed on the external surface of the fibres. However, these molecules will not be as protected as those located inside the pores.

\subsection{Stability of the sepiolite clay in the geopolymer matrix}

In spite of the aggressive alkaline medium in which geopolymerization takes place, sepiolite should be stable in a geopolymer medium for three main reasons. The first is the limited time during which the medium is really aggressive, with $\mathrm{pH}$ values above 14 . This happens in the very first hours of reaction only, when the potassium silicate is in solution. Once setting has occurred, the $\mathrm{pH}$ is reduced to a mild alkaline value of 9 to $10{ }^{25,26}$ The second reason is the well-known, less reactive character of crystalline silicates in geopolymer formation, due to the kinetic difference between the speed of mineral dissolution compared to the speed of geopolymer formation. ${ }^{35}$ The third is the also well-known less reactive character of magnesium silicates (such as sepiolite) as compared to aluminium silicates (such as kaolin) in the geopolymerization reaction. ${ }^{36}$

Table 1 Specific surface area $\left(B E T, N_{2}\right)$ and dye content $(\mathrm{CHN}$ elemental analyses) in sepiolite and sepiolite-dye samples (as: aqueous solution; ss: solid state procedure)

\begin{tabular}{llll}
\hline Sample & $\begin{array}{l}\text { Micropore } \\
\text { volume } \\
\left(\mathrm{cm}^{3} \mathrm{~g}^{-1}\right)\end{array}$ & $\begin{array}{l}\text { Specific } \\
\text { surface area } \\
\left(\mathrm{m}^{2} \mathrm{~g}^{-1}\right)\end{array}$ & $\begin{array}{l}\text { Dye content } \\
(\text { moles } / 100 \mathrm{~g} \\
\text { clay) }\end{array}$ \\
\hline Sepiolite & 0.079 & 340 & 0 \\
Sepiolite $+\mathrm{MB}_{\text {as }}$ & 0.043 & 229 & $5.1 \times 10^{-3}$ \\
Sepiolite $+\mathrm{MR}_{\text {as }}$ & 0.077 & 320 & $1.3 \times 10^{-3}$ \\
Sepiolite $+\mathrm{MB}_{\mathrm{ss}}$ & 0.007 & 114 & $20.2 \times 10^{-3}$ \\
Sepiolite $+\mathrm{MR}_{\mathrm{ss}}$ & 0.007 & 125 & $26.9 \times 10^{-3}$
\end{tabular}

Actually, sepiolite is known to have only a low solubility in alkaline solutions. Treatment for 28 days in severe alkaline conditions produced but small surface structural changes, mainly in external $\mathrm{Si}-\mathrm{OH}$ groups and coordinated water molecules. ${ }^{37}$ Only after a drastic treatment in alkaline conditions does the sepiolite structure break down to form an amorphous and more reactive material. ${ }^{38}$ As a conclusion, the limited attempts to synthesize geopolymers from magnesium silicates showed that a thermal dehydroxylation treatment and a long exposition to alkaline media were required to obtain substantial reactivity ${ }^{39}$ which is not the case in the present study.

In this section, we present additional evidence for the stability of sepiolite in geopolymers from calorimetry measurements. As illustrated by Fig. 4, calorimetric measurements support the stability assumption, as the energy released during geopolymerisation is reduced when metakaolin is replaced by sepiolite in the mix. This means that sepiolite is acting as an inert body in the reaction medium. In addition, the calorimetric measurements confirm the extreme rapidity of the main reaction (dissolution of metakaolinite) as most of the reaction is completed in less than 10 minutes. As it was already discussed sepiolite has a very low solubility in high alkali media ${ }^{37}$ and cannot participate to the geopolymerisation as metakaolin did. After that step, reorganization of the geopolymeric matrix in milder $\mathrm{pH}$ conditions can occur. ${ }^{40}$

In spite of this indirect evidence for sepiolite stability, the XRD patterns show that the intensity of the strong (010) sepiolite diffraction peak around 7.4 degrees $(2 \theta)$ is decreasing due to the dilution effect. As sepiolite is a non-swelling clay ${ }^{41}$ the structural parameters do not change with the insertion of molecular species as methylene blue. ${ }^{12}$ Actually, this peak could only be observed in systems containing large amounts of sepiolite ( $>5 \%$ by weight), while the metakaolin hump is shifted to the position characteristic of aluminosilicate geopolymers (around 30 degrees, 2 $\theta$ ) (Fig. 5 and Table 2). This is significantly different from the position observed in geopolymers prepared from thermally destabilized sepiolite (37 degrees, $2 \theta) .{ }^{39}$ Interestingly, the maximum intensity of the XRD pattern of the geopolymers remained stable even when $10 \%$ of sepiolite was added. This is another proof that sepiolite does not participate in the geopolymerization reaction in our conditions. The partial alteration of the sepiolite in the aggressive conditions of the

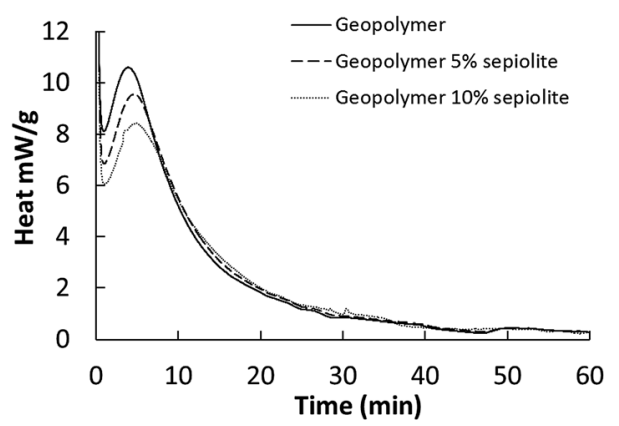

Fig. 4 Reduction of the heat released during the geopolymerisation reaction by addition of sepiolite. 


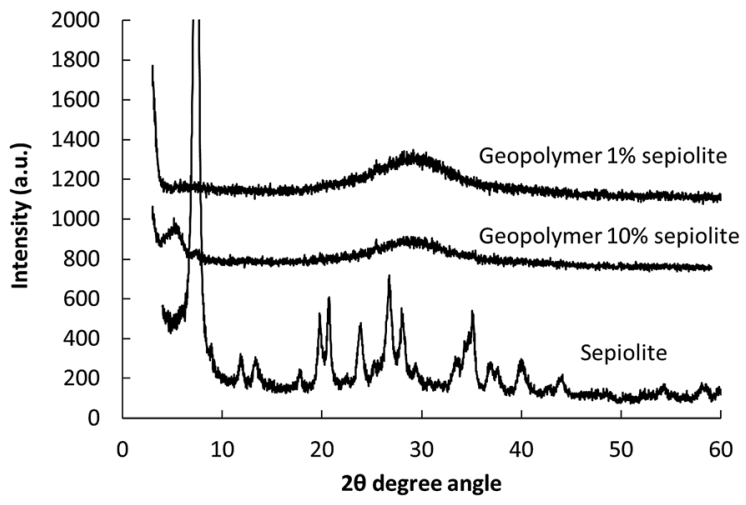

Fig. 5 X-Ray diffraction patterns of pure sepiolite and geopolymers with $1 \%$ and $10 \%$ sepiolite.

Table 2 Measured XRD peak position of geopolymer hump with sepiolite

\begin{tabular}{ll}
\hline$\%$ sepiolite & Peak position $(2 \theta)$ \\
\hline 1 & 29.2 \\
5 & 30.1 \\
10 & 29.2
\end{tabular}

geopolymer formation could still occur; a last indirect evidence of the stability of the sepiolite in the geopolymeric network is the stability of the composite pigment in UV-vis, as detailed in the next sections. This stability seems actually possible only when the dye is encapsulated in the sepiolite that should then remain stable.

\subsection{Stability of the sepiolite-dye composite pigments in the geopolymer matrix}

As pointed out in Section 3.1, the most prominent feature of the UV-vis spectrum of the sepiolite-MB composite pigments is a strong band in the $600-670 \mathrm{~nm}$ range, with a maximum around $670 \mathrm{~nm}$, ascribed to MB monomers encapsulated in sepiolite channels. This signature is maintained when the composite pigment is incorporated in the geopolymer matrix, showing that the composite pigment has not been significantly altered. However, the intensity of the low wavelength side of the band is somewhat decreased, especially with the pigment prepared by the solid-state method (Fig. 6). MB molecules directly incorporated at $0.5 \%$ in the geopolymer exhibit a very different spectrum, with a broad band in the 500-600 nm range ascribed to MB trimers aggregates (Fig. 6). Adding sepiolite in addition to MB leads to the same result.

This shows that MB and sepiolite do not interact with each other when added separately in the geopolymer matrix. Interestingly, at very low MB concentration (0.02\%), the maximum is shifting toward longer wavelengths $(600-650 \mathrm{~nm})$, closer to the position of monomers. This suggests that, at very low concentration, some MB molecules may have been incorporated in the pseudo-zeolitic cavities of the geopolymer network.

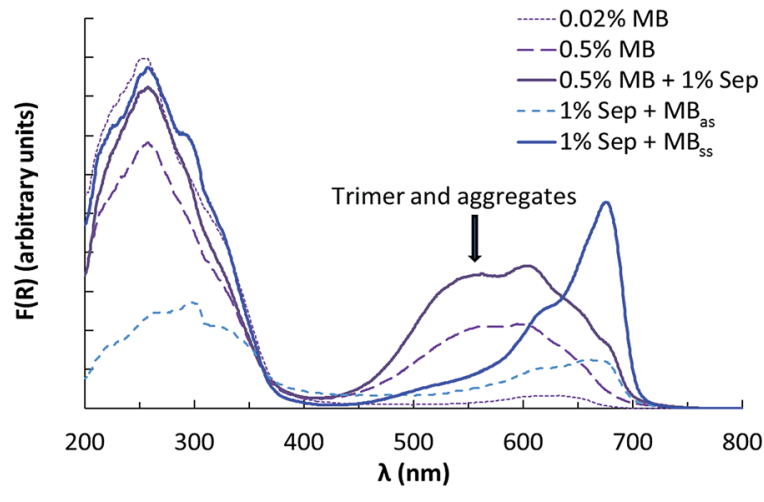

Fig. 6 UV-visible diffuse reflectance spectra of the geopolymers samples after incorporation of methylene blue (0.02 and $0.5 \% \mathrm{MB})$, or a mixture of sepiolite and methylene blue $(0.5 \% \mathrm{MB}+1 \%$ sep), or the composite sepiolite-dye pigment prepared in aqueous solution or by solid-state reaction ( $1 \%$ sep $-M_{\text {as }}$ or $1 \%$ sep $-M_{\text {ss, }}$, respectively).

In parallel to the spectroscopic data, visual inspection of the coloured materials provides evidence for the stability of the composite pigment in the geopolymer matrix. Indeed, the colour of geopolymer samples loaded with the composite pigment is - and remains - blue, just like the composite pigment itself (ESI Fig. $1 \dagger$ ). Conversely, geopolymer samples loaded with MB molecules turn violet, most probably due to the local $\mathrm{pH}$ changes in the geopolymer. A similar behaviour is observed with MR (ESI Fig. $1 \dagger$ ). Geopolymers samples coloured with the composite sepiolite-MR pigment keep the red colour of the dye, whereas samples coloured with MR molecules turn yellow-orange, which is expected as this dye changes its colour in alkaline medium, as also observed in. ${ }^{42}$

\subsection{Resistance to acid and hydrogen peroxide treatments}

The colour of geopolymer samples with the sepiolite-dye composite pigments was found to be significantly more resistant to acid and hydrogen peroxide than samples coloured with the dye. Thus, after $48 \mathrm{~h}$ in a $1 \%$ sulphuric acid solution, the colour change $\Delta E$ (see eqn (1)) of the sample coloured with the composite pigment prepared by the aqueous solution method was only 3.88 , to be compared to 9.9 for the sample coloured with the pure MB dye, at the same total dye loading $(0.2 \%)$. A similar result was obtained when longer (one week) but milder ( $\mathrm{pH}$ 2) treatments were applied to the same type of samples $\Delta E=0.6$ and 2.9, respectively. In the same conditions, the colour changes measured with geopolymer samples incorporating sepiolite-dye pigments prepared by the solid state method show $\Delta E$ values of 1.6 and 1.1 for MB and MR, respectively. Considering the value of the Just Noticeable Difference (JND = 2.3, see Section 2.4), our results show that there is practically no change in colour in the case of geopolymer samples incorporating sepiolite-based pigments.

Another aspect of acid resistance is the resistance of the geopolymer network itself. Geopolymers are known to be acidresistant or at least, more acid-resistant than Portland cement-based materials. ${ }^{27}$ Thus, after one week in $\mathrm{pH} 2$, the mass loss of the geopolymer functionalized with the sepiolite pigment, prepared with either the aqueous solution or the solid 
state method, was $2.4 \%$ and the $\mathrm{pH}$ rose from 10.8 to 12.3 . When the same geopolymer was subsequently exposed to $1 \%$ acid for $48 \mathrm{~h}$, the mass loss due to the acid attack was only $0.6 \%$. The acid resistance could be further improved by optimizing the curing conditions and the aggregate. The resistance of the colour to bleaching by hydrogen peroxide attack was tested by exposing samples for one hour to a $3 \%$ by weight aqueous solution. As qualitatively illustrated in Fig. 7, the colour of geopolymer samples loaded with the composite sepiolite-MB pigment was stable, irrespective of the preparation method ("as" or "ss") (Fig. 7). However, the samples prepared with MR by the solid state method were bleached, as expected from the known easy degradation of this red pigment in hydrogen peroxide. ${ }^{43}$ The geopolymer coloured only with $\mathrm{MB}$ was also bleached. The sepiolite protected the $\mathrm{MB}$ to $\mathrm{H}_{2} \mathrm{O}_{2}$ exposure of the geopolymer. Detailed analysis of the effect of $\mathrm{H}_{2} \mathrm{O}_{2}$ would come at the next step of the research.

\subsection{Resistance to UV exposure}

The stabilization of the colour to UV exposure was determined in two steps: a low UV intensity ( $5 \mathrm{~W}$ ) exposure for $48 \mathrm{~h}$ at $40{ }^{\circ} \mathrm{C}$ and then twice $24 \mathrm{~h}$, also at $40{ }^{\circ} \mathrm{C}$ but at a much higher UV intensity $(800 \mathrm{~W})$. The results corresponding to the cumulative colour change $(\Delta E)$ with respect to the coloured control geopolymer samples unexposed to UV are shown in Fig. 8. When $\mathrm{MB}$ is not incorporated to the sepiolite prior to the geopolymer

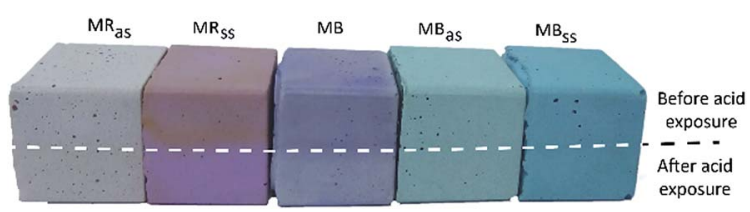

Fig. 7 Change of colour of geopolymer with hydrogen peroxide, from left to right: sepiolite $+M R_{a s}$, sepiolite $+M R_{s s}$, only $M B$, sepiolite + $M B_{a s}$, sepiolite with $\mathrm{MB}_{\text {ss. }}$. Upper part, before $\mathrm{H}_{2} \mathrm{O}_{2}$ exposure and lower part after exposure.

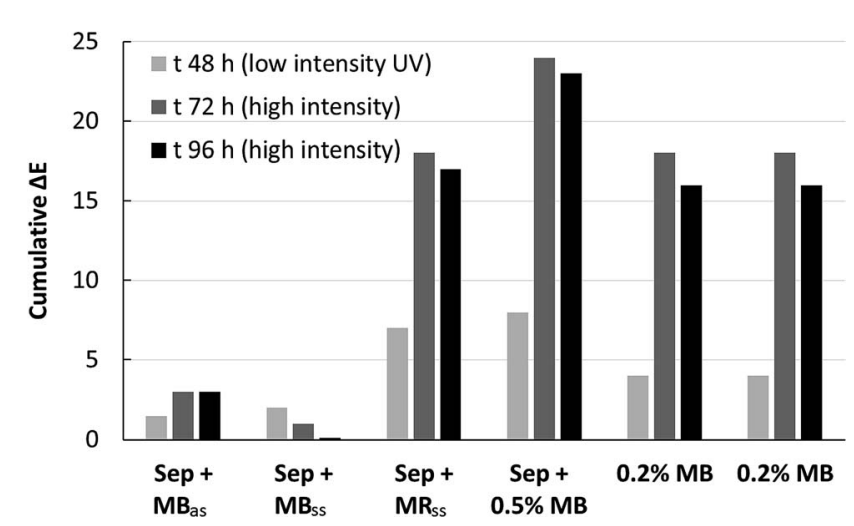

Fig. 8 Cumulative colour change of geopolymer samples coloured with the sepiolite pigment under UV exposure. A similar coloured geopolymer exposed to natural light condition and ambient room temperature is considered as a reference. preparation, the stability of the colour cannot be achieved even with low UV intensity exposure.

This is different from the samples coloured by the sepiolite-dye composite pigment prepared with both methods where colour change differences are not noticeable. The geopolymer coloured with the MR prepared by the solid state method could also not be stabilized and the geopolymers turned pink with UV exposure, which created a greater colour change after 24 hours (Fig. 9). This phenomenon is due to the faster photodegradation kinetics of methyl red under UV. ${ }^{44}$ The colour of the methylene blue dye encapsulated in the sepiolite prepared with the solid state method remains stable after $48 \mathrm{~h}$ of low intensity irradiation, as well as after $48 \mathrm{~h}$ at high intensity irradiation, which confirmed the stability of the Maya blue effect of this preparation.

This is different from the samples coloured by the sepiolite-dye composite pigment prepared with both methods where colour change differences are not noticeable. The geopolymer coloured with the MR prepared by the solid state method could also not be stabilized and the geopolymers turned pink with UV exposure, which created a greater colour change after 24 hours (Fig. 9). This phenomenon is due to the faster photodegradation kinetics of methyl red under UV. ${ }^{44}$ The colour of the methylene blue dye encapsulated in the sepiolite prepared with the solid state method remains stable after $48 \mathrm{~h}$ of low intensity irradiation, as well as after $48 \mathrm{~h}$ at high intensity irradiation, which confirmed the stability of the Maya blue effect of this preparation. The aqueous preparation method seems slightly more sensitive to the high intensity UV exposure than the solid state one even if it is largely more stable than without the incorporation of the dye in the sepiolite.

\subsection{Additional properties provided by sepiolite incorporation in geopolymer}

In addition to the protection of organic dye from the geopolymeric matrix as well as from external aggressive

a)

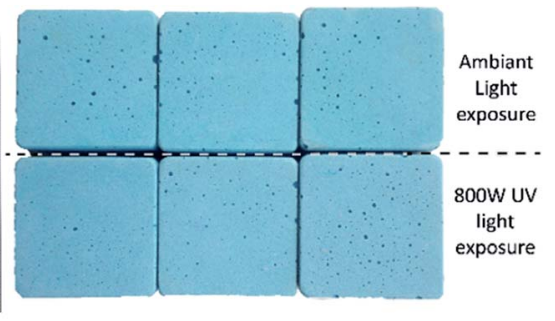

b)

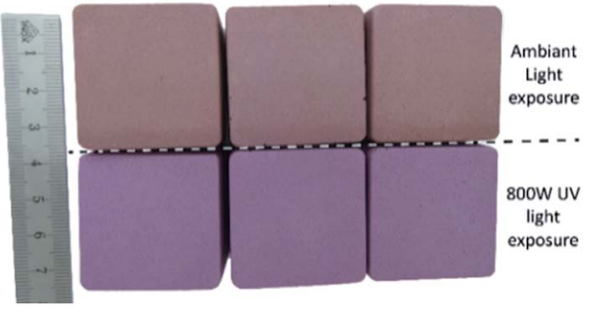

Fig. 9 Geopolymer prepared with the solid state method (a) with MB and (b) with MR, exposed only to ambient light conditions (top), after $48 \mathrm{~h}$ UV exposure at $800 \mathrm{~W}$ (below). Triplets are repetitions. 
environment, it might be worth considering potential benefits for the geopolymeric matrix itself of the sepiolite incorporation. Due to the elongated shape of the sepiolite, it could be used as fibre reinforcement. Mechanical properties of sepiolite have already been determined at different length scales, from macroscale using the universal testing machine, mesoscale using nanoindentation and even individual direct measurement by AFM techniques of single sepiolite microfibers. ${ }^{45}$ However, the tested elastic moduli present considerably differences that are attributed to an effect of the fibers aggregation. ${ }^{45}$

Sepiolite fibers have been used for reinforcement of organic polymers $^{41}$ and a relative increase of the Young modulus by a factor of 2.5 has been observed. However in general, and particularly for stiffer polymers, a much modest reinforcement are observed. ${ }^{41}$ It can be supposed similar behaviour for geopolymer matrices, even if further investigation would be needed to confirm the small effect of sepiolite on mechanical properties. Studies conducted for the use of sepiolite as reinforcement of cementious materials also confirm this weak mechanical improvement. A recent study showed indeed a slight improvement of flexural properties of the cement paste when sepiolite is incorporated. ${ }^{46} \mathrm{~A}$ maximum increase of $5 \%$ of the bending strength was observed when $10 \%$ of sepiolite was added. ${ }^{46}$ This improvement is negligible and can be understood by elastic properties similarities between mineral binders and sepiolite. However, sepiolite addition in very weak binders such as aerated cementitious products have shown to be efficient to reduce micro cracking and significantly improve elastic modulus. ${ }^{47}$ As a consequence for our study, the very small addition of sepiolite in the dense structure of our binder will most probably not influence the flexural strength even if further experiments could be done.

Finally, in addition to the flexural strength, it should also mentioned that sepiolite due to its high aspect ratio has a very strong effect on the rheological behaviour of the paste increasing strongly its apparent viscosity. ${ }^{48}$ As the fresh properties of geopolymeric binders are characterised already by a very high viscosity compared to cementitious binders, ${ }^{49}$ the addition of sepiolite will need to be kept as small as possible, which thus hinder potential mechanical benefits as high content would be needed.

\section{Conclusion}

The novelty of the reported approach is to use synthetic pigments inspired from Maya blue in view of obtaining geopolymers with long lasting colour, resistant to chemical and photochemical aggressions. The synthetic pigments were prepared by encapsulating organic dyes into the structural nanotunnels of sepiolite clay. The pigments were shown to be resistant to the temporary very aggressive alkaline environment encountered during geopolymer synthesis. Furthermore, the pigment-loaded geopolymers were shown to be stable under UV exposure and in mild acidic conditions. The pigment prepared with sepiolite and methylene blue by the solid state method was the most resistant to UV exposure. On a general level, our results validate the concept of double encapsulation - first the organic dye molecule into the clay, then the pigment into the geopolymer matrix - to obtain functionalized and durable geopolymer binders. As geopolymers are emerging as a promising class of cold ceramics and a possible substitute to Portland cement, this opens the way to numerous potential applications.

\section{Acknowledgements}

Financial support from CICYT (Spain; project MAT2012-31759) is gratefully acknowledged. The metakaolin was kindly provided by Imerys. Authors gratefully acknowledge M. A. Camblor for helping in the elaboration of sepiolite-dye schematic models.

\section{References}

1 M. Sanchez Del Rio, P. Martinetto, C. Reyes-Valerio, E. Dooryhée and M. Suarez, Archaeometry, 2006, 48, 115-130. 2 H. van Olphen, Science, 1966, 154, 645-646.

3 R. J. Gettens, Am. Antiq., 1962, 27, 557-564.

4 P. Tagle, A. Alberto, G. Infante de los Reyes, H. Paschinger and H. Richard, Chemical physical characterization of a blue pigment from colonial Havana, ICCROM, 1989.

5 B. Hubbard, W. Kuang, A. Moser, G. A. Facey and C. Detellier, Clays Clay Miner., 2003, 51, 318-326.

6 M. Sánchez del Río, E. Boccaleri, M. Milanesio, G. Croce, W. van Beek, C. Tsiantos, G. Chyssikos, V. Gionis, G. Kacandes, M. Suárez and E. García-Romero, J. Mater. Sci., 2009, 44, 5524-5536.

7 H. Berke, Chem. Soc. Rev., 2007, 36, 15-30.

8 G. Chiari, R. Giustetto, J. Druzik, E. Doehne and G. Ricchiardi, Appl. Phys. A: Mater. Sci. Process., 2008, 90, 3-7.

9 M. José-Yacamán, L. Rendón, J. Arenas and M. C. S. Puche, Science, 1996, 273, 223-225.

10 L. Nicole, C. Laberty-Robert, L. Rozes and C. Sanchez, Nanoscale, 2014, 6, 6267-6292.

11 Ed. E. Galán and A. Singer, Developments in PalygorskiteSepiolite Research, Elsevier, Oxford, 2011.

12 E. Ruiz-Hitzky, J. Mater. Chem., 2001, 11, 86-91.

13 E. Ruiz-Hitzky, P. Aranda, A. Álvarez, J. Santarén and A. Esteban-Cubillo, in Developments in Clay Science, ed. G. Emilio and S. Arieh, Elsevier, 2011, vol. 3, pp. 393-452.

14 E. Ruiz-Hitzky, M. Darder, F. M. Fernandes, B. Wicklein, A. C. S. Alcântara and P. Aranda, Prog. Polym. Sci., 2013, 38, 1392-1414.

15 R. Kleber, L. Masschelein-Kleiner and J. Thissen, Stud. Conserv., 1967, 12, 41-56.

16 D. E. Arnold, J. R. Branden, P. R. Williams, G. M. Feinman and J. P. Brown, Antiquity, 2007, 82, 151-164.

17 A. Doménech, M. T. Doménech-Carbó and H. G. M. Edwards, J. Raman Spectrosc., 2011, 42, 86-96.

18 R. Giustetto, O. Wahyudi, I. Corazzari and F. Turci, Appl. Clay Sci., 2011, 52, 41-50.

19 A. J. Aznar, B. Casal, E. Ruiz-Hitzky, I. Lopez-Arbeloa, F. Lopez-Arbeloa, J. Santaren and A. Alvarez, Clay Miner., 1992, 27, 101-108.

20 S. Ovarlez, F. Giulieri, F. Delamare, N. Sbirrazzuoli and A.-M. Chaze, Microporous Mesoporous Mater., 2011, 142, 371-380. 
21 N. Volle, Ph.D. thesis, Nice-Sophia-Antipolis - UFR Sciences, 2011.

22 N. Volle, L. Challier, A. Burr, F. Giulieri, S. Pagnotta and A.-M. Chaze, Compos. Sci. Technol., 2011, 71, 1685-1691.

23 I. Amato, Nature, 2013, 494, 300-301.

24 J. Davidovits, J. Therm. Anal., 1991, 37, 1633-1656.

25 Ed. J. L. Provis and J. S. J. van Deventer, Alkali Activated Materials: State-of-the-Art Report, RILEM TC 224-AAM, Springer, Heidelberg, 2014.

26 P. Duxson, A. Fernández-Jiménez, J. L. Provis, G. C. Lukey, A. Palomo and J. S. J. Deventer, J. Mater. Sci., 2007, 42, 2917-2933.

27 T. Bakharev, Cem. Concr. Res., 2005, 35, 658-670.

28 J. Temuujin, W. Rickard, M. Lee and A. van Riessen, J. NonCryst. Solids, 2011, 357, 1399-1404.

29 K. J. D. MacKenzie and B. O'Leary, Mater. Lett., 2009, 63, 230232.

30 D. Saha, P. Parimita Sahoo, G. Madras and T. N. Guru Row, RSC Adv., 2012, 2, 10505-10511.

31 J. J. Koenderick, Color for the Sciences, The MIT Press, Cambridge MA, 2010.

32 G. Sharma, in Digital Color Imaging Handbook, ed. G. Sharma, CRC Press, New York, 2002.

33 K. Y. Jacobs and R. A. Schoonheydt, Langmuir, 2001, 17, 5150-5155.

34 R. Garcia Moreno, D. Strivay and B. Gilbert, J. Raman Spectrosc., 2008, 39, 1050-1056.

35 H. Xu and J. S. J. van Deventer, Int. J. Miner. Process., 2000, 59, 247-266.

36 A. F. White and S. L. Brantley, Rev. Mineral. Geochem., 1995, 31, 1-22.
37 S. Martinez-Ramirez, F. Puertas and M. T. Blanco-Varela, Clay Miner., 1996, 31, 225-232.

38 D. C. Golden, J. B. Dixon, H. Shadfan and L. A. Kippenberger, Clays Clay Miner., 1985, 33, 44-50.

39 K. D. MacKenzie, S. Bradley, J. Hanna and M. Smith, J. Mater. Sci., 2013, 48, 1787-1793.

40 M. S. Muñiz-Villarreal, A. Manzano-Ramírez, S. SampieriBulbarela, J. R. Gasca-Tirado, J. L. Reyes-Araiza, J. C. Rubio-Ávalos, J. J. Pérez-Bueno, L. M. Apatiga, A. Zaldivar-Cadena and V. Amigó-Borrás, Mater. Lett., 2011, 65, 995-998.

41 E. Ruiz-Hitzky, P. Aranda, M. Darder and F. M. Fernandes, in Developments in Clay Science, ed. B. Faïza and L. Gerhard, Elsevier, 2013, vol. 5, pp. 721-741.

42 V. F. F. Barbosa and K. J. D. MacKenzie, Mater. Res. Bull., 2003, 38, 319-331.

43 M. Zhou, Q. Yu, L. Lei and G. Barton, Sep. Purif. Technol., 2007, 57, 380-387.

44 C. Guillard, H. Lachheb, A. Houas, M. Ksibi, E. Elaloui and J.-M. Herrmann, J. Photochem. Photobiol., A, 2003, 158, 2736.

45 F. M. Fernandes, L. Vazquez, E. Ruiz-Hitzky, A. Carnicero and M. Castro, RSC Adv., 2014, 4, 11225-11231.

46 T. Kavas, E. Sabah and M. S. Çelik, Cem. Concr. Res., 2004, 34, 2135-2139.

47 A. Sepulcre Aguilar, J. Pinilla Melo and F. Hernández Olivares, Construct. Build. Mater., 2013, 47, 282-292.

48 L. Martinie, P. Rossi and N. Roussel, Cem. Concr. Res., 2010, 40, 226-234.

49 A. Favier, J. Hot, G. Habert, N. Roussel and J.-B. d'Espinose de Lacaillerie, Soft Matter, 2014, 10, 1134-1141. 